\title{
Precise analysis of compressive strain effects on electric power generation properties of a lead zirconate titanate piezoelectric ceramic
}

\author{
Mitsuhiro OKAYASU ${ }^{a,}{ }^{*}$, Keisuke WATANABE $^{b}$ \\ ${ }^{a}$ Graduate School of Natural Science and Technology, Okayama University, 3-1-1 Tsushimanaka, Kita-ku, \\ Okayama, 700-8530, Japan \\ ${ }^{b}$ Graduate School of Science and Engineering, Ehime University, 3 Bunkyo-cho, Matsuyama, \\ Ehime, 790-8577, Japan
}

Received: June 28, 2015; Revised: August 18, 2015; Accepted: August 25, 2015

(C) The Author(s) 2015. This article is published with open access at Springerlink.com

\begin{abstract}
Electric power generation characteristics of lead zirconate titanate (PZT) piezoelectric ceramic have been examined during cyclic loading with different compressive strain value. A thin PZT ceramic plate attached to a thin brass plate was used. With an increasing compressive strain value in the PZT ceramic plate, the electric voltage increased, and the highest electric voltage was obtained for the sample with the high strain level $(0.4 \%)$. The electric voltage decreased for the sample with more than $0.45 \%$ strain because of the failure in the PZT ceramic. The electric voltage was different depending on the strain condition, where the higher strain and the wider strain range $(\Delta \varepsilon)$ made the high electric voltage. Moreover, the electric voltage was attributed to the strain rate, where the higher strain rate made higher electric voltage due to high kinetic energy. The electric generation characteristics could be estimated with an operation of the strain value and strain ratio $\left(\varepsilon_{\min } / \varepsilon_{\max }\right)$ especially for the sample loaded cyclically under the slow loading speed. This was affected by the linear correlation between the electric voltage and the strain value. Based upon the experimental work, suitable loading condition to make high electric voltage could be proposed.
\end{abstract}

Keywords: energy harvesting; piezoelectric ceramic; cyclic loading; electric power generation

\section{Introduction}

For environmental issues, development of clean energy system is required in our society. There are several clean energy systems, such as wind power, wave power, solar power, and geothermal power. In addition, functional materials have also received special attention in our society, e.g., piezoelectric ceramics. Piezoelectric ceramics have the ability to transform mechanical strain

* Corresponding author.

E-mail: mitsuhiro.okayasu@utoronto.ca energy into electrical charge (piezoelectric effect) or applied electrical energy into mechanical strain. With piezoelectric effect, an attempt is made to create energy harvesting systems. One of the main piezoelectric materials consists of lead zirconate titanate $\left(\mathrm{PbZrTiO}_{3}\right.$, PZT) ceramic formed by a perovskite structure.

Piezoelectric energy harvesters have been produced as vibrating devices, which convert mechanical strain energy into electricity [1]. Using a number of PZT ceramic plates set in parallel under a floor in stadiums and train stations, energy harvesting devices have been proposed. As the energy harvester generates limited 
electrical energy, e.g., a few milliwatts [2], power management is a crucial consideration. The piezoelectric properties to create electric power generation have been given significant interest for use in harvesting elements. To make high electric power generation from PZT ceramic, a discussion on the associated research has been presented [3-7]. In particular, the influence of the vibrating condition on the electric power generation characteristics has been investigated experimentally using commercial PZT ceramic plates, where cyclic loading, loading frequency, and strain value were altered $[8,9]$. Although several investigators have examined electric power generation characteristics using piezoelectric ceramics, there appears to be little data concerning the effects of strain condition on the electric power generation. Since the strain value and strain behavior directly contribute to the electric power generation characteristics, that information is significantly important to design a high efficiency energy harvesting system. Hence, in the present work, the effects of the strain condition on the electric power generation characteristics were examined using the PZT ceramic and our original testing systems.

\section{Experimental procedures}

In the present work, the commercial circular membrane PZT $\left(\mathrm{PbZrTiO}_{3}\right)$ ceramic was selected. The PZT ceramic, formed with dimension of $\phi=9.0 \mathrm{~mm}$ and $0.12 \mathrm{~mm}$ in thickness, was attached to a thin brass plate with dimension of $\phi=12.0 \mathrm{~mm}$ and $0.10 \mathrm{~mm}$ in thickness. Silver-based electrode platting was conducted on the PZT ceramic surface. The electric wires were connected directly on the electrode and the brass plate by solder. The nominal grain size of this PZT examined was about $1 \mu \mathrm{m}$. The PZT ceramic consisted of a tetragonal structure with an aspect ratio $c / a=1.014$.

(a)
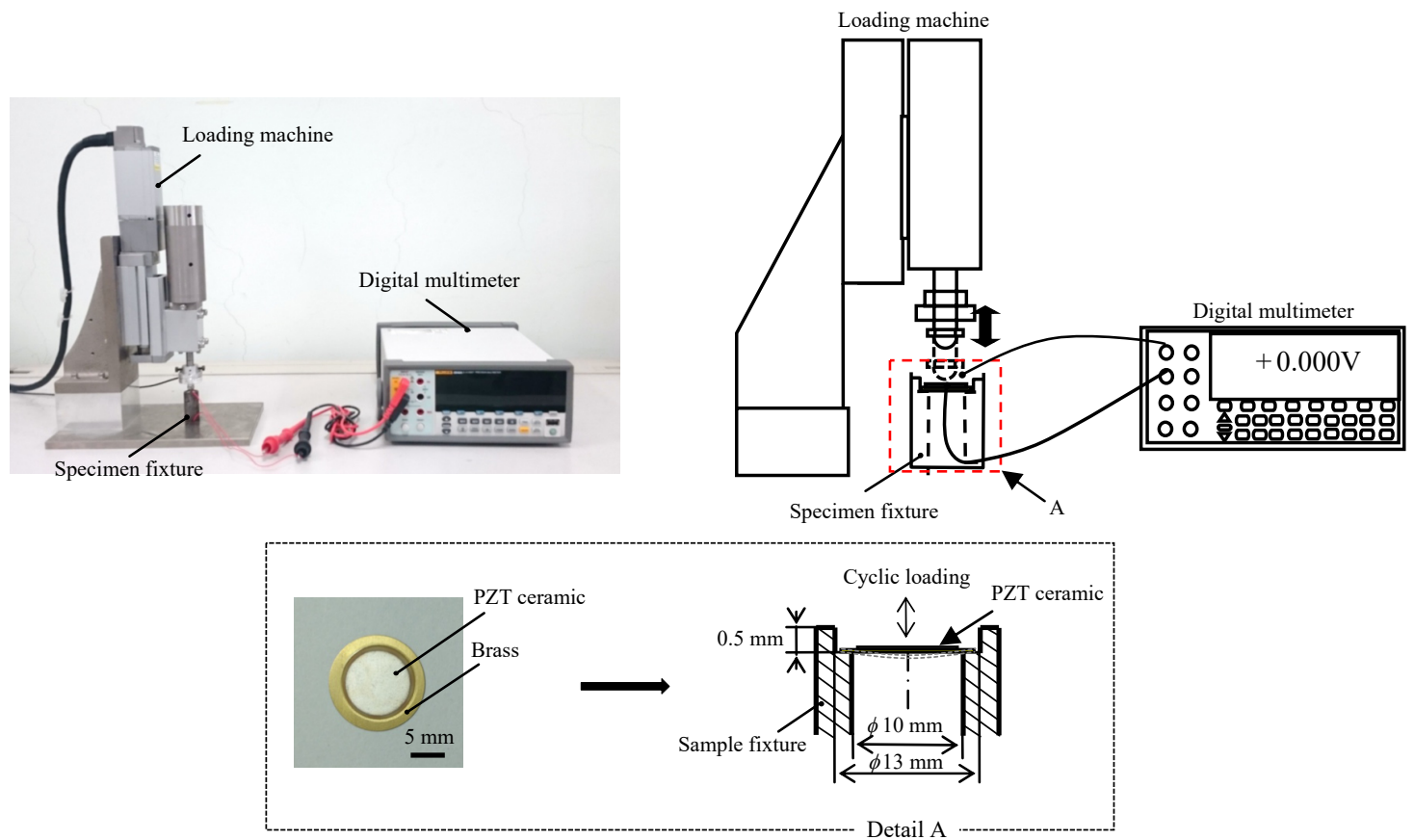

(b)

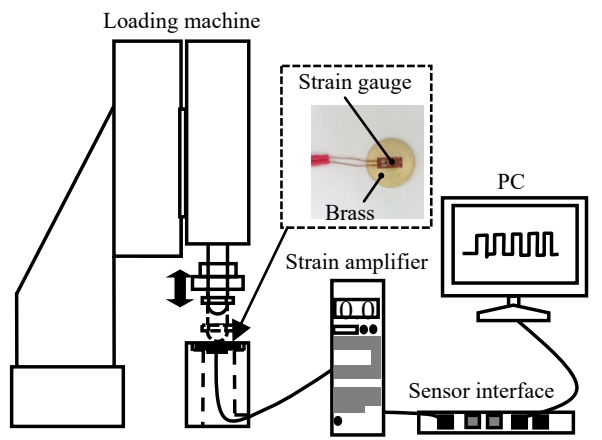

Fig. 1 PZT ceramic plate, experimental setup, and testing methods for electric power generation tests. 
Figure 1 shows schematic illustrations and photographs of the test apparatus and testing methods. In this approach, the electric power generation characteristics from PZT ceramic were investigated under different strain conditions, e.g., different strain value $(\varepsilon=0.1 \%-1.1 \%)$, strain range $(\Delta \varepsilon=0-0.4 \%)$, strain ratio $\left(S R=\varepsilon_{\min } / \varepsilon_{\max }=0-0.63\right)$, and load waveform (square or triangular waveform). The applied load was conducted using round rod (semicircular face) to the center of the PZT ceramic. Electric power generation (the maximum mean electric voltage) was examined during cyclic loading for 10 cycles. Note that long loading time to the PZT ceramic may make change of the electric power generation characteristics, so the short cyclic number of 10 was selected as the first approach. The electric voltage generated from the PZT ceramic during the cyclic loading was monitored continuously using a digital multimeter (8846A, Fluke).

\section{Results and discussion}

Figure 2 shows the variation of the electric voltage with time for different $\varepsilon, \Delta \varepsilon$, and $S R$ values under square and triangular waveforms. As seen, the maximum electric voltage value and the increment rate of the electric voltage for square wave mode are overall higher than those of the triangular wave one under the same strain condition due to the high kinetic energy for square wave mode. A sharp peak voltage is obtained for all square waveforms and decreases suddenly to $0 \mathrm{~V}$ even if the applied load is conducted. This occurrence may give a reduction in the electrons in the PZT ceramic. With the removal of the applied load, a reversal of the electrons to the PZT ceramic occurs, leading to the generation of the negative electric voltage. With increasing the strain range $\Delta \varepsilon$ in both wave modes, the peak of electric voltage increases apparently. Even though the high strain is applied to the PZT ceramic (conditions (d) and (e): $\varepsilon_{\max }=0.25 \%$ and $0.4 \%$ respectively) in both wave modes, the electric voltage variation is shifted to the lower level compared to that for condition (c): $\varepsilon_{\max }=$ $0.4 \%$ ). From this, it is considered that strain range $\Delta \varepsilon$ could severely contribute to the electric power generation. To understand the electric power generation characteristics more clearly, the results of Fig. 2 are further analyzed.

Figure 3 shows the maximum electric voltage values obtained under different strain conditions. It is verified that the electric voltage value obtained for square wave mode is overall higher than that for triangular wave mode. Moreover, the higher strain or the higher strain range $(\Delta \varepsilon)$ makes the high electric voltage. It should be noted that the loading condition (a) $(\Delta \varepsilon=0.1 \%)$ makes lower electric voltage compared to that for the sample loaded with the similar $\Delta \varepsilon$ value (conditions (d) and (e)). Because of the different strain ratio $\left(S R, \varepsilon_{\min } / \varepsilon_{\max }\right)$, the factor of $S R$ could strongly affect the electric power generation compared to $\Delta \varepsilon$.

With the results of Fig. 3(a), an attempt was made to estimate the electric voltage with an operation of the electric voltage values. As first approximation, the strain value was added with different patterns to make the same $\Delta \varepsilon$ level, e.g., strain values are $0.25 \%$ and $0.4 \%$ under $S R=0$ (see Fig. 3(b)). Interestingly, the electric voltage values are found to be almost similar under the same $\Delta \varepsilon$ level for the sample loaded under triangular wave mode, e.g., the electric voltages of about $10 \mathrm{~V}$ and $15 \mathrm{~V}$ for the $\Delta \varepsilon$ values $0.25 \%$ and $0.4 \%$ respectively. On the contrary, no clear correlation is obvious for the results of square wave mode. Such a well-estimation of the $\Delta \varepsilon$ value for the triangular wave mode would be affected by the slow loading speed, as shown in Fig. 2, where there would have a linear correlation between the strain and electric voltage value. On the other hand, rapid increment of the strain and electric voltage value for square wave mode (Fig. 2) may not have linear correlation.

Effect of strain value on the maximum electric voltage was further investigated and the obtained results are shown in Fig. 4. The electric voltage for square wave mode is overall higher than that for triangular one, which is a consequence of high energy arising from the high loading speed. In both wave modes, the electric voltage increases with increasing the strain value, although it then decreases after more than $0.4 \%$ and $0.7 \%$ strain values for square and triangular wave modes, respectively. The peak point for square wave mode at $0.4 \%$ strain is about $40 \%$ higher than that for triangular one. The reason behind this is also the high applied energy, where the sample failure might have occurred [8].

\section{Conclusions}

Electric power generation characteristics of PZT 
Square wave mode

$S R$ : strain ratio $\left(\varepsilon_{\min } / \varepsilon_{\max }\right)$

\begin{tabular}{|l|l|l|l|l|l|l|}
\hline (a) $\varepsilon_{\max }=0.1 \%, \Delta \varepsilon=0.1 \%, S R=0$ & (b) $\varepsilon_{\max }=0.25 \%, \Delta \varepsilon=0.25 \%, S R=0$ & (c) $\varepsilon_{\max }=0.4 \%, \Delta \varepsilon=0.4 \%, S R=0$ \\
\hline
\end{tabular}

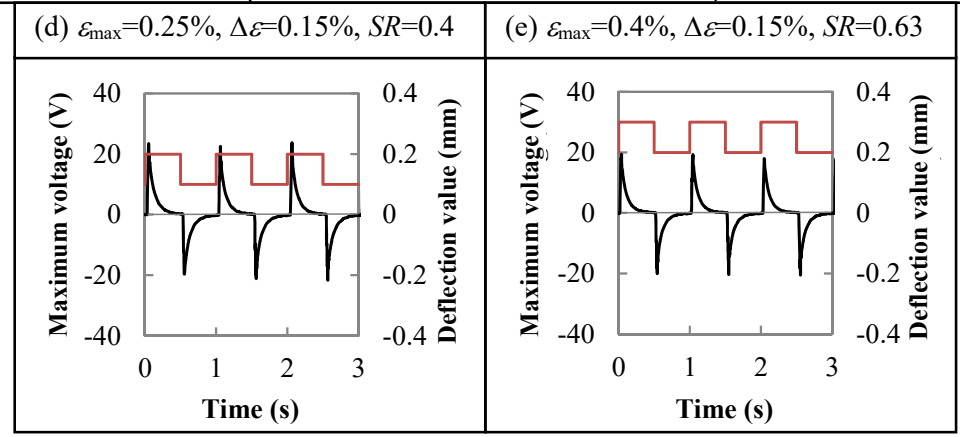

Triangular wave mode

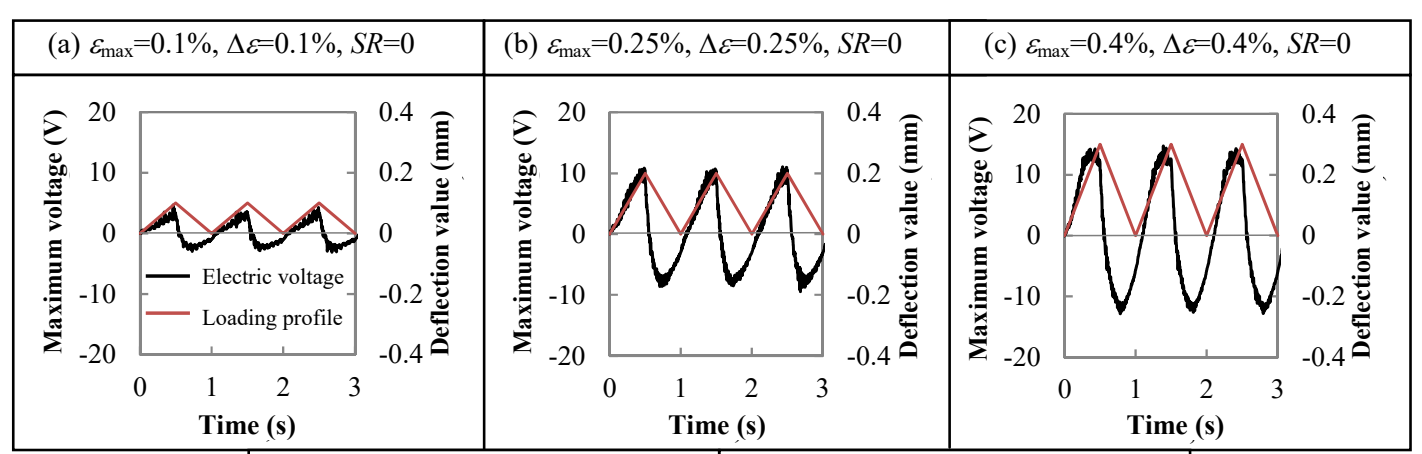

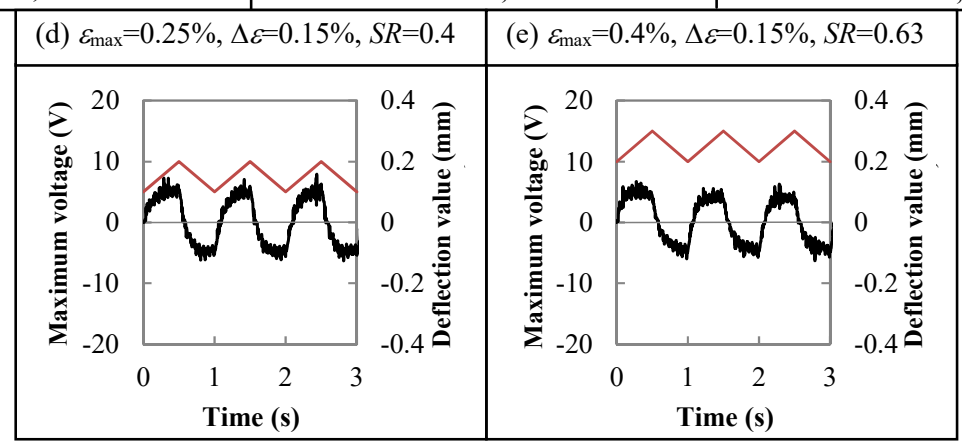

Fig. 2 Variation of electric voltage with time for the PZT ceramic plate under square and triangular wave modes.

piezoelectric ceramic plate have been examined experimentally. In this approach, various cyclic loading conditions were applied to the PZT ceramic plate to make high efficiency energy harvesting system. Based on the above results and discussion, the following conclusions can be drawn.
(1) With an increasing strain value in the PZT ceramic plate, the electric voltage increases with the highest electric voltage being obtained for the sample with $0.4 \%$ strain. The electric voltage decreases when the strain occurs severely due to the damage in the PZT ceramic plate. 


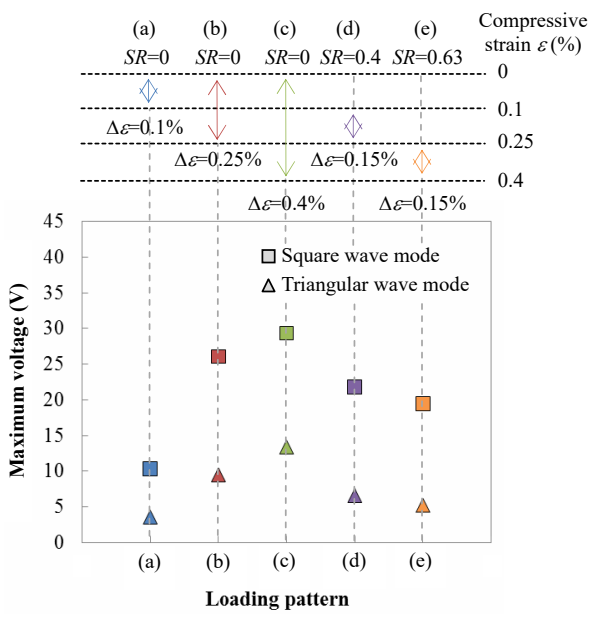

(a) Different strain conditions

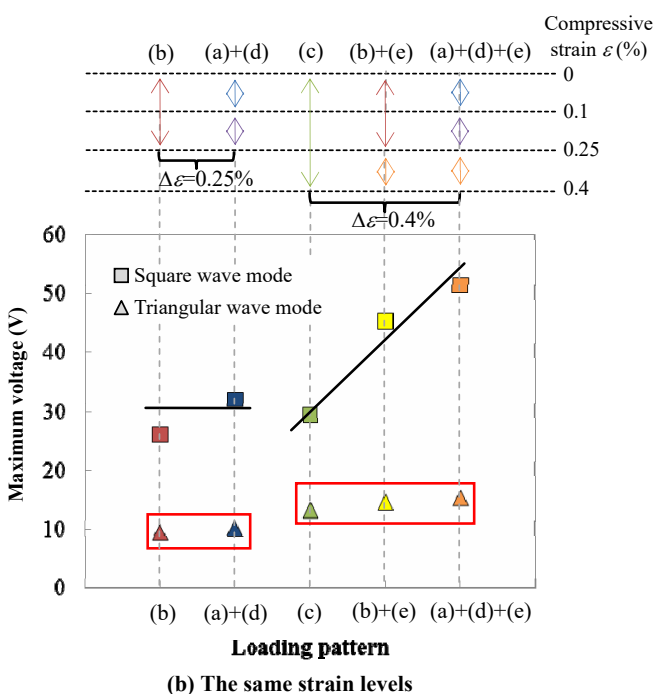

(b) The same strain levels

Fig. 3 Variation of the mean maximum electric voltage as a function of the cyclic frequency: (a) different strain conditions and (b) the same strain levels.

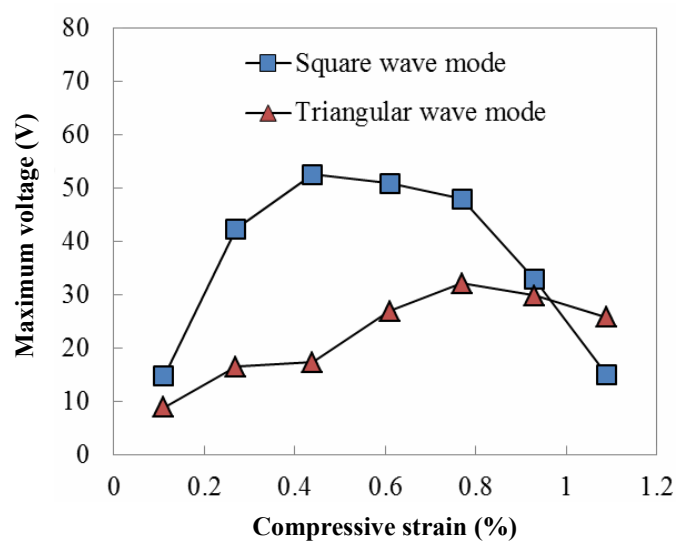

Fig. 4 Variation of the mean maximum electric voltage as a function of the strain value obtained under square and triangular wave modes.
(2) The electric voltage is also dependent on the strain range $(\Delta \varepsilon)$, where the higher strain range makes the higher electric voltage. The maximum electric voltage can be estimated well with the $\Delta \varepsilon$ value and strain ratio for the sample as loaded under the triangular wave mode.

(3) The electric voltage is influenced by the cyclic loading condition and material failure in the PZT ceramic. The high electric voltage is obtained for the square wave mode because of the high kinetic energy. Based upon the experimental work, appropriate loading condition to make high electric voltage could be proposed.

\section{References}

[1] Tabesh A, Frechette LG. A low-power stand-alone adaptive circuit for harvesting energy from a piezoelectric micropower generator. IEEE $T$ Ind Electron 2010, 57: 840-849.

[2] Chen H, Jia C, Zhang C, et al. Power harvesting with PZT ceramics. In Proceedings of IEEE International Symposium on Circuits and Systems, 2007: 557-560.

[3] Shu YC, Lien IC. Analysis of power output for piezoelectric energy harvesting systems. Smart Mater Struct 2006, 15: 1499-1512.

[4] Kim HW, Priya S, Uchino K, et al. Piezoelectric energy harvesting under high pre-stressed cyclic vibrations. $J$ Electroceram 2005, 15: 27-34.

[5] Poulin G, Sarraute E, Costa F. Generation of electrical energy for portable devices: Comparative study of an electromagnetic and a piezoelectric system. Sensor Actuat A: Phys 2004, 116: 461-471.

[6] Sudevalayam S, Kulkarni P. Energy harvesting sensor nodes: Survey and implications. IEEE Communications Surveys \& Tutorials 2011, 13: 443-461.

[7] Lefeuvre E, Seald G, Guyomar D, et al. Materials, structures and power interfaces for efficient piezoelectric energy harvesting. $J$ Electroceram 2009, 22: 171-179.

[8] Okayasu M, Sato D, Sato Y, et al. A study of the effects of vibration on the electric power generation properties of lead zirconate titanate piezoelectric ceramic. Ceram Int 2012, 38: 4445-4451.

[9] Okayasu M, Mizuno M, Shiraishi T. Characteristics of electrical power generation from lead zirconate titanate piezoelectric ceramics under cyclic loading. Ceram Int 2014, 40: 6589-6595.

Open Access The articles published in this journal are distributed under the terms of the Creative Commons Attribution 4.0 International License (http://creativecommons.org/licenses/ by/4.0/), which permits unrestricted use, distribution, and reproduction in any medium, provided you give appropriate credit to the original author(s) and the source, provide a link to the Creative Commons license, and indicate if changes were made. 